SISSA REF. 193/93/A

DFPD $93 / \mathrm{A} / 80$

December 1993

\title{
THE THREE-POINT CORRELATION FUNCTION OF THE COSMIC MICROWAVE BACKGROUND IN INFLATIONARY MODELS
}

\author{
Alejandro Gangui ${ }^{1}$, Francesco Lucchin $^{2}$, Sabino Matarrese ${ }^{3}$ and Silvia Mollerach ${ }^{4}$ \\ ${ }^{1}$ SISSA - International School for Advanced Studies, \\ via Beirut 2 - 4, 34013 Trieste, Italy \\ ${ }^{2}$ Dipartimento di Astronomia Università di Padova, \\ vicolo dell'Osservatorio 5, 35122 Padova, Italy \\ ${ }^{3}$ Dipartimento di Fisica "Galileo Galilei" Università di Padova, \\ via Marzolo 8, 35131 Padova, Italy \\ ${ }^{4}$ Theory Division CERN, CH-1211, Genève 23, Switzerland
}

Submitted to The Astrophysical Journal

\begin{abstract}
We analyze the temperature three-point correlation function and the skewness of the Cosmic Microwave Background (CMB), providing general relations in terms of multipole coefficients. We then focus on applications to large angular scale anisotropies, such as those measured by the COBE DMR, calculating the contribution to these quantities from primordial, inflation generated, scalar perturbations, via the Sachs-Wolfe effect. Using the techniques of stochastic inflation we are able to provide a universal expression for the ensemble averaged three-point function and for the corresponding skewness, which accounts for all primordial second-order effects. These general expressions would moreover apply to any situation where the bispectrum of the primordial gravitational potential has a hierarchical form. Our results are then specialized to a number of relevant models: power-law inflation driven by an exponential potential, chaotic inflation with a quartic and quadratic potential and a particular case of hybrid inflation. In all these cases non-Gaussian effects are small: as an example, the mean skewness is much smaller than the cosmic rms skewness implied by a Gaussian temperature fluctuation field.
\end{abstract}




\section{Introduction}

The recent detection of large-scale Cosmic Microwave Background (CMB) anisotropies by $C O B E$ (Smoot et al. 1992) has provided us with a unique opportunity to probe the properties of the primordial perturbations which affect the CMB temperature distribution through the Sachs-Wolfe effect (Sachs \& Wolfe 1967). Future years of observation will reduce the level of the noise, thus making possible to extract more and more statistical information from the data. Besides the classical analysis in terms of the rms temperature fluctuation on the smoothing scale of the DMR instrument, the angular two-point correlation function and the lowest order multipoles (Smoot et al. 1992; Wright et al. 1992), preliminary results have already appeared on a measurement of the three-point function and skewness on the $C O B E$ map (Hinshaw et al. 1993; Smoot et al. 1993). The three-point correlation function of CMB fluctuations, and its limit at zero angular separation, the skewness, provide an estimate of the size of possible deviations from a Gaussian behaviour of the primordial perturbation field.

There are at present two main theories on the origin of primordial perturbations, namely that they originated from quantum fluctuations of scalar fields during an early inflationary era or from topological defects produced by a phase transition in the early universe. Present observational data have not yet been able to rule out one of them. It was generally thought that the main difference between the two models is the statistical distribution of the resulting fluctuations: inflationary models were claimed to predict a Gaussian distribution, while topological defects a non-Gaussian one. However, it is now clear that, at least for the cosmic string case, even if the effect of a single string is clearly non-Gaussian, the combined effect of a large number of them results in a quasi-Gaussian distribution. On the other hand, it has been realized that inflationary models also predict small deviations from Gaussianity. Hence, it is necessary to make more quantitative estimates of the non-Gaussian features expected in each model if one intends to use the predicted statistical distribution of CMB anisotropies to discriminate between them.

Besides instrumental noise and contamination by Galactic emission, any analysis of largescale anisotropies is however limited by one more source of indetermination: the so-called "cosmic variance" (Abbott \& Wise 1984; Smoot et al. 1992), namely the impossibility of making observations in more than one universe, which severely limits our ability to extract intrinsic non-Gaussian signals from the data, especially for anisotropy patterns dominated by low-order multipoles (Scaramella \& Vittorio 1991). This is particularly important when considering the temperature three-point correlation function of the $C O B E$ map, since most present theoretical models of the primordial density fluctuation process predict rather small non-Gaussian effects on such large scales, i.e. a quasi-Gaussian anisotropy pattern. Actually, the large beam-width of the $C O B E$ DMR experiment blurs any information on smaller scales, which makes it practically insensitive to the type of non-Gaussian signatures produced by topological defects. Cosmic strings predict relevant non-Gaussian features in the CMB at angular scales below a few arcminutes (Moessner, Perivolaropoulos \& Brandenberger 1993). This makes even more interesting obtaining a quantitative prediction on the non-Gaussian CMB features which are expected to be produced on very large angular scales in inflationary models. In fact, every sensible inflation model will produce some small but non-negligible non-Gaussian effects, both by the self-interaction of the inflaton field, and by the local 
back-reaction of its self-gravity. It is our interest here to quantify this prediction, with the largest possible generality, so that observational results on the CMB angular three-point function can be used as a further test on the nature of the primordial perturbation process.

Unfortunately, we will find that single-field inflation models generally imply mean values for the skewness which are well below the cosmic rms skewness of a Gaussian field, which confirms and generalizes earlier results based on a simple toy-model (Falk, Rangarajan \& Srednicki 1993; Srednicki 1993). In this sense, looking at anisotropies on smaller angular scales than $C O B E$ would probably appear a more promising strategy. Coulson, Pen \& Turok (1993) and Coulson et al. (1993) consider degree-scale anisotropies produced by nonGaussian field-ordering theories of structure formation. The results of the present paper would also apply to these intermediate scales, only provided the window function appropriate to the specific experiment is used.

Falk et al. (1993) first gave a quantitative estimate of the size of non-Gaussian effects through a calculation of the three-point CMB correlation function from perturbations generated in a simple model, where the inflaton has cubic self-interactions. Luo \& Schramm (1993) argued that secondary anisotropies could also play a non-negligible role in this respect.

Our paper is the first one where the problem is considered in a totally general and self-consistent way. We use the stochastic approach to inflation (e.g. Starobinskii 1986; Goncharov, Linde \& Mukhanov 1987), as a technical tool to self-consistently account for all second-order effects in the generation of scalar field fluctuations during inflation and their giving rise to curvature perturbations. We also properly account for the non-linear relation between the inflaton fluctuation and the peculiar gravitational potential. Our derivation moreover removes a non-realistic restriction to purely de Sitter background made by Falk et al. (1993), which is especially important when non-Zel'dovich perturbation spectra are considered.

The plan of the paper is as follows. In Section 2 we give completely general definitions for the CMB three-point function in a single sky in terms of multipole coefficients. We stress that these results actually apply to whichever type of temperature fluctuations, as well as to any angular scale. We also give what we believe is a more operational definition of angular three-point function than has been considered so far (e.g. Luo 1993a,b). In the last part of this section we anticipate the results obtained from the Sachs-Wolfe effect due to the quasi-Gaussian perturbations of the gravitational potential generated during inflation and we discuss the effects of the cosmic variance on the possibility of observing a non-zero mean value for the skewness. As a by-product of this analysis we compute the "dimensionless" rms skewness for a large range of the primordial spectral index of density fluctuations $n$. All the results reported at this level only depend on three quantities: i) the rms quadrupole amplitude $\mathcal{Q}$, which is fixed by observational data; ii) a model-dependent parameter $\Phi_{3} \sim$ a few, related to the amplitude of the skewness; iii) the rotationally invariant multipole coefficients $\mathcal{C}_{\ell}$ (suitably normalized to the quadrupole, $\ell=2$ ), whose specific dependence on $\ell$ is fixed by the spectral index $n$. Note that inflation is able to produce perturbation spectra with essentially all values of $n$ around unity (e.g. Mollerach, Matarrese \& Lucchin 1993), as it can be needed to match the COBE data (Smoot et al. 1992) with observations on smaller scales. The technical derivation in the frame of stochastic inflation of the connected two- and three-point function (or its Fourier counterpart, the bispectrum), for the inflaton 
field first and the local gravitational potential next, is reported in Section 3. Section 4 deals with the zero-lag limit of the three-point function: the skewness, for which we provide a universal inflationary expression, which we then specialize to some of the most popular inflaton potentials: exponential (Lucchin \& Matarrese (1985)), quartic and quadratic (Linde 1983, 1985) as well as a simple potential for hybrid inflation (Linde 1993). Section 5 contains some general conclusions.

\section{The CMB three--point correlation function}

Our aim here is to compute the CMB temperature three-point correlation function implied by possible departures from a Gaussian behaviour of the primordial peculiar gravitational potential. As a first step we will define the connected two- and three-point correlation functions of the CMB temperature as measured by a given observer, i.e. on a single microwave sky. Let us then define the temperature fluctuation field $\frac{\Delta T}{T}(\vec{x} ; \hat{\gamma}) \equiv\left(T(\vec{x} ; \hat{\gamma})-T_{0}(\vec{x})\right) / T_{0}(\vec{x})$, where $\vec{x}$ specifies the position of the observer, the unit vector $\hat{\gamma}$ points in a given direction from $\vec{x}$ and $T_{0}(\vec{x}) \equiv \int \frac{d \Omega_{\hat{\gamma}}}{4 \pi} T(\vec{x} ; \hat{\gamma})$ represents the mean temperature of the CMB measured by that observer (i.e. the monopole term).

The angular two-point correlation function $C_{2}(\vec{x} ; \alpha)$ measured by an observer placed in $\vec{x}$ is the average product of temperature fluctuations in two directions $\hat{\gamma}_{1}$ and $\hat{\gamma}_{2}$ whose angular separation is $\alpha$; this can be written as

$$
C_{2}(\vec{x} ; \alpha)=\int \frac{d \Omega_{\hat{\gamma}_{1}}}{4 \pi} \int \frac{d \Omega_{\hat{\gamma}_{2}}}{2 \pi} \delta\left(\hat{\gamma}_{1} \cdot \hat{\gamma}_{2}-\cos \alpha\right) \frac{\Delta T}{T}\left(\vec{x} ; \hat{\gamma}_{1}\right) \frac{\Delta T}{T}\left(\vec{x} ; \hat{\gamma}_{2}\right) .
$$

As well known, in the limit $\alpha \rightarrow 0$ one recovers the CMB variance $C_{2}(\vec{x})=\int \frac{d \Omega_{\hat{\gamma}}}{4 \pi}\left[\frac{\Delta T}{T}(\vec{x} ; \hat{\gamma})\right]^{2}$. Expanding the temperature fluctuation in spherical harmonics

$$
\frac{\Delta T}{T}(\vec{x} ; \hat{\gamma})=\sum_{\ell=1}^{\infty} \sum_{m=-\ell}^{\ell} a_{\ell}^{m}(\vec{x}) \mathcal{W}_{\ell} Y_{\ell}^{m}(\hat{\gamma}),
$$

and writing the Dirac delta function as a completeness relation for Legendre polynomials $P_{\ell}$, we easily arrive at the expression

$$
C_{2}(\vec{x} ; \alpha)=\frac{1}{4 \pi} \sum_{\ell} P_{\ell}(\cos \alpha) Q_{\ell}^{2}(\vec{x}) \mathcal{W}_{\ell}^{2},
$$

where $Q_{\ell}^{2}=\sum_{m=-\ell}^{\ell}\left|a_{\ell}^{m}\right|^{2}$. In the previous expressions $\mathcal{W}_{\ell}$ represents the window function of the specific experiment. Setting $\mathcal{W}_{0}=\mathcal{W}_{1}=0$ automatically accounts for both monopole and dipole subtraction; for $\ell \geq 2$ one can take $\mathcal{W}_{\ell} \simeq \exp \left[-\frac{1}{2} \ell(\ell+1) \sigma^{2}\right]$, where $\sigma$ is the dispersion of the antenna-beam profile, which measures the angular response of the detector (e.g. Wright et al. 1992). In some cases the quadrupole term is also subtracted from the maps (e.g. Smoot et al. 1992); in this case we also set $\mathcal{W}_{2}=0$.

The analogous expression for the angular three-point correlation function is obtained by taking the average product of temperature fluctuations in three directions $\hat{\gamma}_{1}, \hat{\gamma}_{2}$ and $\hat{\gamma}_{3}$ with fixed angular separations $\alpha$ (between $\hat{\gamma}_{1}$ and $\hat{\gamma}_{2}$ ), $\beta$ (between $\hat{\gamma}_{2}$ and $\hat{\gamma}_{3}$ ) and $\gamma$ (between $\hat{\gamma}_{1}$ 
and $\hat{\gamma}_{3}$ ); these angles have to satisfy the obvious inequalities $|\alpha-\gamma| \leq \beta \leq \alpha+\gamma$. One then has

$$
\begin{aligned}
& C_{3}(\vec{x} ; \alpha, \beta, \gamma)=\int \frac{d \Omega_{\hat{\gamma}_{1}}}{4 \pi} \int_{0}^{2 \pi} \frac{d \varphi_{12}}{2 \pi} \int_{-1}^{1} d \cos \vartheta_{12} \delta\left(\cos \vartheta_{12}-\cos \alpha\right) \int_{-1}^{1} d \cos \vartheta_{23} \\
& \quad \times \delta\left(\cos \vartheta_{23}-\cos \beta\right) \int_{-1}^{1} d \cos \vartheta_{13} \delta\left(\cos \vartheta_{13}-\cos \gamma\right) \frac{\Delta T}{T}\left(\vec{x} ; \hat{\gamma}_{1}\right) \frac{\Delta T}{T}\left(\vec{x} ; \hat{\gamma}_{2}\right) \frac{\Delta T}{T}\left(\vec{x} ; \hat{\gamma}_{3}\right),
\end{aligned}
$$

where $\cos \vartheta_{\alpha \beta} \equiv \hat{\gamma}_{\alpha} \cdot \hat{\gamma}_{\beta}$ and $\varphi_{12}$ is the azimuthal angle of $\hat{\gamma}_{2}$ on the plane orthogonal to $\hat{\gamma}_{1}$. The above relation can be rewritten in a form analogous to Eq.(四), namely

$$
\begin{aligned}
& C_{3}(\vec{x} ; \alpha, \beta, \gamma)=N(\alpha, \beta, \gamma) \int \frac{d \Omega_{\hat{\gamma}_{1}}}{4 \pi} \int \frac{d \Omega_{\hat{\gamma}_{2}}}{2 \pi} \int \frac{d \Omega_{\hat{\gamma}_{3}}}{2} \delta\left(\hat{\gamma}_{1} \cdot \hat{\gamma}_{2}-\cos \alpha\right) \\
& \quad \times \delta\left(\hat{\gamma}_{2} \cdot \hat{\gamma}_{3}-\cos \beta\right) \delta\left(\hat{\gamma}_{1} \cdot \hat{\gamma}_{3}-\cos \gamma\right) \frac{\Delta T}{T}\left(\vec{x} ; \hat{\gamma}_{1}\right) \frac{\Delta T}{T}\left(\vec{x} ; \hat{\gamma}_{2}\right) \frac{\Delta T}{T}\left(\vec{x} ; \hat{\gamma}_{3}\right),
\end{aligned}
$$

where $N(\alpha, \beta, \gamma) \equiv \sqrt{1-\cos ^{2} \alpha-\cos ^{2} \beta-\cos ^{2} \gamma+2 \cos \alpha \cos \beta \cos \gamma}$. . Setting $\alpha=\beta=\gamma=0$ in these general expressions one obtains the CMB skewness $C_{3}(\vec{x})=\int \frac{d \Omega_{\hat{\gamma}}}{4 \pi}\left[\frac{\Delta T}{T}(\vec{x} ; \hat{\gamma})\right]^{3}$. Also useful are the equilateral three-point correlation function (e.g. Falk et al. 1993) and the collapsed one (e.g. Hinshaw et al. 1993 and references therein), corresponding to the choices $\alpha=\beta=\gamma$, and $\alpha=\gamma, \beta=0$, respectively. Alternative statistical estimators, more suited to discriminate bumpy non-Gaussian signatures in noisy data, have been recently introduced by Graham et al. (1993). In all the above formulas, full-sky coverage was assumed, for simplicity. The effects of partial sky coverage on some of the statistical quantities considered here are discussed in detail by Scott, Srednicki \& White (1993).

Following the procedure used above for $C_{2}(\vec{x} ; \alpha)$, we can rewrite the three-point function in the form

$$
\begin{aligned}
& C_{3}(\vec{x} ; \alpha, \beta, \gamma)=N(\alpha, \beta, \gamma) \frac{\pi}{2} \sum_{\ell_{1}, \ell_{2}, \ell_{3}} \sum_{m_{1}, m_{2}, m_{3}} a_{\ell_{1}}^{m_{1}} a_{\ell_{2}}^{m_{2}} a_{\ell_{3}}^{m_{3} *} \mathcal{W}_{\ell_{1}} \mathcal{W}_{\ell_{2}} \mathcal{W}_{\ell_{3}} \\
& \quad \times \sum_{j, k, \ell} \sum_{m_{j}, m_{k}, m_{\ell}} P_{j}(\cos \alpha) P_{k}(\cos \beta) P_{\ell}(\cos \gamma) \mathcal{H}_{j \ell \ell_{1}}^{m_{j} m_{\ell} m_{1}} \mathcal{H}_{k j \ell_{2}}^{m_{k} m_{j} m_{2}} \mathcal{H}_{k \ell \ell_{3}}^{m_{k} m_{\ell} m_{3}}
\end{aligned}
$$

where the coefficients $\mathcal{H}_{\ell_{1} \ell_{2} \ell_{3}}^{m_{1} m_{2} m_{3}} \equiv \int d \Omega_{\hat{\gamma}} Y_{\ell_{1}}^{m_{1} *}(\hat{\gamma}) Y_{\ell_{2}}^{m_{1}}(\hat{\gamma}) Y_{\ell_{3}}^{m_{3}}(\hat{\gamma})$, which can be easily expressed in terms of Clebsch-Gordan coefficients (e.g. Messiah 1976), are only non-zero if the indices $\ell_{i}, m_{i}(i=1,2,3$,$) fulfill the relations: \left|\ell_{j}-\ell_{k}\right| \leq \ell_{i} \leq\left|\ell_{j}+\ell_{k}\right|, \ell_{1}+\ell_{2}+\ell_{3}=$ even and $m_{1}=m_{2}+m_{3}$. The collapsed three-point function measured by the observer in $\vec{x}$ reads

$$
C_{3}(\vec{x} ; \alpha)=\frac{1}{4 \pi} \sum_{\ell_{1}, \ell_{2}, \ell_{3}} \sum_{m_{1}, m_{2}, m_{3}} P_{\ell_{1}}(\cos \alpha) a_{\ell_{1}}^{m_{1}} a_{\ell_{2}}^{m_{2}} a_{\ell_{3}}^{m_{3} *} \mathcal{W}_{\ell_{1}} \mathcal{W}_{\ell_{2}} \mathcal{W}_{\ell_{3}} \mathcal{H}_{\ell_{3} \ell_{2} \ell_{1}}^{m_{3} m_{2} m_{1}}
$$

which, for $\alpha=0$, gives a useful expression for the skewness.

So far our expressions have been kept completely general, they would apply to whatever source of temperature fluctuations in the sky, through suitable (usually statistical) relations

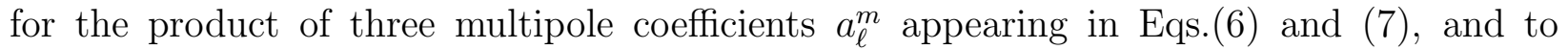
whatever angular scale, through the specific choice of window functions $\mathcal{W}_{\ell}$. However, in

\footnotetext{
${ }^{1}$ To show that the latter expression is correctly normalized one can expand the delta functions in Legendre polynomials and use the relation $\sum_{\ell}(2 \ell+1) P_{\ell}(x) P_{\ell}(y) P_{\ell}(z)=\frac{2}{\pi}\left(1-x^{2}-y^{2}-z^{2}+2 x y z\right)^{-1 / 2}$.
} 
what follows we shall only deal with large angular scale anisotropies originated from primary perturbations in the gravitational potential $\Phi$ on the last scattering surface via the SachsWolfe effect (Sachs \& Wolfe 1967). In that case $\frac{\Delta T}{T}(\vec{x} ; \hat{\gamma})=\frac{1}{3} \Phi\left(\vec{x}+r_{0} \hat{\gamma}\right)$, where $r_{0}=2 / H_{0}$ is the horizon distance and $H_{0}$ the Hubble constant. As noticed by Luo \& Schramm (1993), secondary anisotropies produced during the mildly non-linear evolution of perturbations through the Rees-Sciama effect (Rees \& Sciama 1968; Martínez-González, Sanz \& Silk 1992), also imply a non-zero contribution to the connected three-point function, which is still there when the underlying primordial linear gravitational potential is Gaussian. Furthermore, these authors suggest that this contribution should strongly dominate over the primary inflationary one. However, a more detailed analysis (Mollerach et al., in preparation) shows that this is not the case on the angular scales probed by the COBE DMR. Therefore, in what follows we will only refer to the primary contribution to the three-point function. A recent analysis of the three-point function and the skewness of the $C O B E$ data has been performed by Hinshaw et al. (1993) and Smoot et al. (1993), who find no statistical evidence for nonGaussian signatures beyond those implied by the cosmic variance: while consistent with the random-phase hypothesis, these data can only rule out strongly non-Gaussian fluctuations on very large scales. On smaller scales, Graham et al. (1993), analyzing the UCSB SP91 experiment, were able to detect non-Gaussian features, which, however, might also be of non-cosmological origin.

To obtain definite predictions for the statistics described above, one needs to exploit the random nature of the multipole coefficients $a_{\ell}^{m}$. In our case, these coefficients should be considered as zero-mean non-Gaussian random variables whose statistics should in principle be deduced from that of the gravitational potential. In such a case one should either obtain, by Monte-Carlo simulations, different realization of the sky corresponding to different "cosmic observers", or analytically compute theoretical ensemble expectation values. The latter procedure will be followed here to obtain the mean two- and three-point functions. These expectation values are of course observer-i.e. $\vec{x}$-independent and can only depend upon the needed number of angular separations.

In the frame of the inflationary model, the calculations reported in the following section lead to general expressions for the mean two- and three-point functions of the primordial gravitational potential, namely

$$
\left\langle\Phi\left(r_{0} \hat{\gamma}_{1}\right) \Phi\left(r_{0} \hat{\gamma}_{2}\right)\right\rangle=\frac{9 \pi \mathcal{Q}^{2}}{5(2 \pi)^{2}} \sum_{\ell \geq 0}(2 \ell+1) P_{\ell}\left(\hat{\gamma}_{1} \cdot \hat{\gamma}_{2}\right) \mathcal{C}_{\ell}
$$

and

$$
\begin{aligned}
& \left\langle\Phi\left(r_{0} \hat{\gamma}_{1}\right) \Phi\left(r_{0} \hat{\gamma}_{2}\right) \Phi\left(r_{0} \hat{\gamma}_{3}\right)\right\rangle=\frac{81 \pi^{2} \mathcal{Q}^{4}}{25(2 \pi)^{4}} \Phi_{3} \sum_{j, \ell \geq 0}(2 j+1)(2 \ell+1) \mathcal{C}_{j} \mathcal{C}_{\ell} \\
& \quad \times\left[P_{j}\left(\hat{\gamma}_{1} \cdot \hat{\gamma}_{3}\right) P_{\ell}\left(\hat{\gamma}_{1} \cdot \hat{\gamma}_{2}\right)+P_{j}\left(\hat{\gamma}_{2} \cdot \hat{\gamma}_{1}\right) P_{\ell}\left(\hat{\gamma}_{2} \cdot \hat{\gamma}_{3}\right)+P_{j}\left(\hat{\gamma}_{3} \cdot \hat{\gamma}_{1}\right) P_{\ell}\left(\hat{\gamma}_{3} \cdot \hat{\gamma}_{2}\right)\right],
\end{aligned}
$$

where $\Phi_{3}$ is a model-dependent coefficient. The $\ell$-dependent coefficients $\mathcal{C}_{\ell}$ are defined by $\left\langle Q_{\ell}^{2}\right\rangle \equiv \frac{(2 \ell+1)}{5} \mathcal{Q}^{2} \mathcal{C}_{\ell}$, with $\mathcal{Q}=\left\langle Q_{2}^{2}\right\rangle^{1 / 2}$ the rms quadrupole, and are related to the gravitational potential power-spectrum $P_{\Phi}(k)$ through $\mathcal{C}_{\ell}=\int_{0}^{\infty} d k k^{2} P_{\Phi}(k) j_{\ell}^{2}\left(k r_{0}\right) / \int_{0}^{\infty} d k k^{2} P_{\Phi}(k) j_{2}^{2}\left(k r_{0}\right)$, where $j_{\ell}$ is the $\ell$-th order spherical Bessel function.2 The rms quadrupole is simply related

\footnotetext{
${ }^{2}$ The possible infrared divergence of this expression for $\ell=0$ has no practical effect on observable quantities, since the monopole is always removed.
} 
to the quantity $Q_{r m s-P S}$ defined by Smoot et al. (1992): $\mathcal{Q}=\sqrt{4 \pi} Q_{r m s-P S} / T_{0}$. For the scales of interest we can make the approximation $P_{\Phi}(k) \propto k^{n-4}$, where $n$ corresponds to the primordial index of density fluctuations (e.g. $n=1$ is the Zel'dovich, scale-invariant case), in which case (e.g. Bond \& Efstathiou 1987; Fabbri, Lucchin \& Matarrese 1987)

$$
\mathcal{C}_{\ell}=\frac{\Gamma\left(\ell+\frac{n}{2}-\frac{1}{2}\right) \Gamma\left(\frac{9}{2}-\frac{n}{2}\right)}{\Gamma\left(\ell+\frac{5}{2}-\frac{n}{2}\right) \Gamma\left(\frac{3}{2}+\frac{n}{2}\right)}
$$

The equations above allow to compute the angular spectrum,

$$
\left\langle a_{\ell_{1}}^{m_{1}} a_{\ell_{2}}^{m_{2} *}\right\rangle=\delta_{\ell_{1} \ell_{2}} \delta_{m_{1} m_{2}} \frac{\mathcal{Q}^{2}}{5} \mathcal{C}_{\ell_{1}}
$$

and the angular bispectrum,

$$
\left\langle a_{\ell_{1}}^{m_{1}} a_{\ell_{2}}^{m_{2}} a_{\ell_{3}}^{m_{3} *}\right\rangle=\frac{3 \mathcal{Q}^{4}}{25} \Phi_{3}\left[\mathcal{C}_{\ell_{1}} \mathcal{C}_{\ell_{2}}+\mathcal{C}_{\ell_{2}} \mathcal{C}_{\ell_{3}}+\mathcal{C}_{\ell_{3}} \mathcal{C}_{\ell_{1}}\right] \mathcal{H}_{\ell_{3} \ell_{1} \ell_{2}}^{m_{3} m_{1} m_{2}}
$$

Replacing the latter expression into Eq.(6) we obtain the general form of the mean threepoint correlation function. Some simplifications occur for the collapsed three-point function, for which we obtain

$$
\begin{aligned}
& \left\langle C_{3}(\alpha)\right\rangle=\frac{3 \mathcal{Q}^{4}}{25(4 \pi)^{2}} \Phi_{3} \sum_{\ell_{1}, \ell_{2}, \ell_{3}}\left(2 \ell_{1}+1\right)\left(2 \ell_{2}+1\right)\left(2 \ell_{3}+1\right) P_{\ell_{1}}(\cos \alpha)\left[\mathcal{C}_{\ell_{1}} \mathcal{C}_{\ell_{2}}+\mathcal{C}_{\ell_{2}} \mathcal{C}_{\ell_{3}}+\mathcal{C}_{\ell_{3}} \mathcal{C}_{\ell_{1}}\right] \\
& \times \mathcal{W}_{\ell_{1}} \mathcal{W}_{\ell_{2}} \mathcal{W}_{\ell_{3}} \mathcal{F}_{\ell_{1} \ell_{2} \ell_{3}}
\end{aligned}
$$

where the coefficients $\mathcal{F}_{\ell_{1} \ell_{2} \ell_{3}} \equiv(4 \pi)^{-2} \int d \Omega_{\hat{\gamma}} \int d \Omega_{\hat{\gamma}^{\prime}} P_{\ell_{1}}\left(\hat{\gamma} \cdot \hat{\gamma}^{\prime}\right) P_{\ell_{2}}\left(\hat{\gamma} \cdot \hat{\gamma}^{\prime}\right) P_{\ell_{3}}\left(\hat{\gamma} \cdot \hat{\gamma}^{\prime}\right)$ may be suitably expressed in terms of products of factorials of $\ell_{1}, \ell_{2}$ and $\ell_{3}$, using standard relations for Clebsch-Gordan coefficients, by noting that $\mathcal{F}_{k \ell m}=\left(\begin{array}{ccc}k & \ell & m \\ 0 & 0 & 0\end{array}\right)^{2}$ (cf. Messiah 1976). The CMB mean skewness $\left\langle C_{3}(0)\right\rangle$ immediately follows from the above equation for $\alpha=0$. A plot of the angular dependence of the collapsed three-point function above, normalized to the skewness, is reported in Figure 1, for typical values of the spectral index $n$.

It was first realized by Scaramella \& Vittorio (1991) that detecting a non-zero three-point function or skewness for temperature fluctuations in the sky cannot be directly interpreted as a signal for intrinsically non-Gaussian perturbations. In fact, even a Gaussian perturbation field has non-zero chance to produce a non-Gaussian sky pattern. This problem is related to what is presently known as cosmic variance, and is particularly relevant for fluctuations on large angular scales, i.e. for low-order multipoles of the temperature fluctuation field. One way to quantify this effect is through the $r m s$ skewness of a Gaussian field $\left\langle C_{3}^{2}(0)\right\rangle_{\text {Gauss }}^{1 / 2}$. It is easy to find

$$
\left\langle C_{3}^{2}(0)\right\rangle_{\text {Gauss }}=3 \int_{-1}^{1} d \cos \alpha\left\langle C_{2}(\alpha)\right\rangle^{3}
$$

Scaramella \& Vittorio (1991) and, more recently, Srednicki (1993), focused on the most popular case of a scale-invariant spectrum, $n=1$ and the COBE DMR window function, corresponding to a Gaussian with dispersion $\sigma=3^{\circ} .2$ (e.g. Wright et al. 1992). We will be interested here in the same quantity, but for various values of $n$. In Figure 2 we have 
plotted the normalized $r m s$ skewness $\left\langle C_{3}^{2}(0)\right\rangle_{\text {Gauss }}^{1 / 2} /\left\langle C_{2}(0)\right\rangle^{3 / 2}$ as a function of the spectral index, both including and removing the quadrupole: in both cases this ratio is in the range $0.1-0.3$ for interesting values of $n$. The values obtained for $n=1$, both with and without the $\ell=2$ contribution, are identical to those given by Srednicki (1993), who adopted the same smoothing angle (the slightly different definition of window function cannot affect our dimensionless skewness ratios). As a rough criterion, we can conclude that, in order to detect primordial non-Gaussian signatures, $\left\langle C_{3}(0)\right\rangle$ must be at least of the same order as $\left\langle C_{3}^{2}(0)\right\rangle_{\text {Gauss }}^{1 / 2}$. On the other hand, as we will see below, single-scalar-field inflationary models generally lead to skewness ratios $\left\langle C_{3}(0)\right\rangle /\left\langle C_{2}(0)\right\rangle^{3 / 2} \lesssim 10^{-4}$, so that their non-Gaussian features cannot be distinguished from the cosmic rms skewness. In this sense the quasiGaussian inflationary predictions for the CMB anisotropies are in full agreement with the recent analysis of the three-point function and the skewness from COBE data (Hinshaw et al. 1993; Smoot et al. 1993).

\section{Stochastic inflation and the statistics of the gravita- tional potential}

Now we will show the validity of Eq.(9) for the three-point correlation function of the gravitational potential due to perturbations produced during an inflationary epoch in the early universe. This calculation will provide the primordial power-spectrum, reflecting into the $\ell$ dependence of the $\mathcal{C}_{\ell}$ coefficients, as well as a general expression for the factors $\mathcal{Q}$ and $\Phi_{3}$ above. In order to take into account all the effects contributing to a non-vanishing primordial three-point correlation function of $\Phi$, we will perform the computation in two steps. In Section 3.1 we compute the three-point function for the inflaton field perturbation $\delta \phi$. The most convenient way to perform this calculation is in the frame of the stochastic approach to inflation (Starobinskii 1986, Goncharov et al. 1987), which naturally takes into account all the multiplicative effects in the inflaton dynamics that are responsible for the non-Gaussian features. Then, in Section 3.2 we compute the extra-contribution to the three-point function of the gravitational potential that arises due to the non-linear relation between $\Phi$ and $\delta \phi$. This effect has been previously noticed by Barrow \& Coles (1990) and Yi \& Vishniac (1993).

\subsection{The inflaton bispectrum}

To study the dynamics of the inflaton, we will apply the stochastic approach. This is based on defining a coarse-grained inflaton field $\phi(\vec{x}, \alpha)$, obtained by suitable smoothing of the original quantum field over a scale larger than the Hubble radius size, whose dynamics is described by a multiplicative Langevin-type equation. This is obtained by adding to the classical equation of motion a Gaussian noise term whose amplitude is fixed by the rms fluctuation of the scalar field at Hubble radius crossing,

$$
\frac{\partial \phi(\vec{x}, \alpha)}{\partial \alpha}=-\frac{V^{\prime}(\phi)}{\kappa^{2} V(\phi)}+\frac{H(\phi)}{2 \pi} \eta(\vec{x}, \alpha),
$$


where $V(\phi)$ is the inflaton potential, primes denote differentiation with respect to $\phi$ and $\kappa \equiv \sqrt{8 \pi G}=\sqrt{8 \pi} / m_{P}$, with $m_{P}$ the Planck mass. The Hubble parameter here should be consistently calculated from the local energy density of the coarse-grained inflaton. The noise term $\eta$ has zero mean and autocorrelation function (e.g. Mollerach et al. 1991)

$$
\left\langle\eta(\vec{x}, \alpha) \eta\left(\vec{x}^{\prime}, \alpha^{\prime}\right)\right\rangle=\hbar j_{0}\left(q_{s}(\alpha)\left|\vec{x}-\vec{x}^{\prime}\right|\right) \delta\left(\alpha-\alpha^{\prime}\right) .
$$

The use of the time variable $\alpha=\ln \left(a / a_{*}\right)$ in this equation has been motivated by Starobinskii (1986), who noticed that $\alpha$ accounts for the possible time dependence of the Hubble parameter. This is particularly relevant when general, i.e. non-de Sitter, inflation is studied. We also defined the coarse-grained domain size through the comoving wave-number $q_{s}(\alpha) \equiv \epsilon H(\alpha) a(\alpha)$, with $\epsilon$ a number smaller than one, $H(\alpha) \equiv H\left(\phi_{c l}(\alpha)\right)$, with $\phi_{c l}(\alpha)$ the homogeneous classical solution of the Langevin equation (i.e., that obtained with the noise term "switched" off). Finally the scale-factor $a(\alpha)$ is obtained by integration of $H(\alpha)$.

The stochastic dynamics of the coarse-grained field within a single coarse-graining domain (i.e., for $\vec{x}=\vec{x}^{\prime}$ ) can be studied in terms of the Fokker-Planck equation for the probability distribution function of $\phi$. In our case, instead, since we are interested also in spatial correlations of the field, we will solve directly the Langevin equation above. To the aim of computing the three-point function of $\phi$, a second-order perturbative expansion around the classical solution is enough. We will require that the potential $V(\phi)$ is a smooth function of its argument, which translates into requiring well defined values for the steepness of the potential $X(\alpha) \equiv X\left(\phi_{c l}(\alpha)\right) \equiv m_{P} V^{\prime}\left(\phi_{c l}\right) / V\left(\phi_{c l}\right)$ and its derivatives (Turner 1993) throughout the range of relevant scales. Apart from this requirement we keep the analysis general; only at the end we will apply our results to some specific inflationary potentials. We first expand $V(\phi)$ around $\phi_{c l}$, up to second order in $\delta \phi(\vec{x}, \alpha) \equiv \phi(\vec{x}, \alpha)-\phi_{c l}(\alpha)$, (i.e. up to order $\hbar$ ), $V(\phi)=V\left(\phi_{c l}\right)+V^{\prime}\left(\phi_{c l}\right) \delta \phi+\frac{1}{2} V^{\prime \prime}\left(\phi_{c l}\right) \delta \phi^{2}+\cdots$. Replacing this into the Langevin equation we obtain

$$
\frac{\partial \delta \phi(\vec{x}, \alpha)}{\partial \alpha}=A(\alpha) \delta \phi(\vec{x}, \alpha)+B(\alpha) \delta \phi^{2}(\vec{x}, \alpha)+\left[D_{1}(\alpha)+D_{2}(\alpha) \delta \phi(\vec{x}, \alpha)\right] \eta(\vec{x}, \alpha)
$$

where we have used $\partial \phi_{c l} / \partial \alpha=-V^{\prime}\left(\phi_{c l}\right) / \kappa^{2} V\left(\phi_{c l}\right)$. In Eq. (17) we defined

$$
A=-\frac{m_{P}}{8 \pi} X^{\prime} ; B=-\frac{m_{P}}{16 \pi} X^{\prime \prime} ; D_{1}=\frac{H(\alpha)}{2 \pi} ; D_{2}=\frac{H(\alpha) X}{4 \pi m_{P}} .
$$

Let us now split the field perturbation as $\delta \phi=\delta \phi_{1}+\delta \phi_{2}$ of $\mathcal{O}\left(\hbar^{1 / 2}\right)$ and $\mathcal{O}(\hbar)$, respectively. We can also define a rescaled variable $\tilde{\eta} \equiv(H(\alpha) / 2 \pi) \eta$. We then find

$$
\begin{aligned}
& \delta \phi_{1}(\vec{x}, \alpha)=X(\alpha) \int_{0}^{\alpha} d \alpha^{\prime} X^{-1}\left(\alpha^{\prime}\right) \tilde{\eta}\left(\vec{x}, \alpha^{\prime}\right) \\
& \delta \phi_{2}(\vec{x}, \alpha)=X(\alpha) \int_{0}^{\alpha} d \alpha^{\prime}\left[\frac{B\left(\alpha^{\prime}\right)}{X\left(\alpha^{\prime}\right)} \delta \phi_{1}^{2}\left(\vec{x}, \alpha^{\prime}\right)+\frac{1}{2 m_{P}} \delta \phi_{1}\left(\vec{x}, \alpha^{\prime}\right) \tilde{\eta}\left(\vec{x}, \alpha^{\prime}\right)\right] .
\end{aligned}
$$

Let us now calculate the connected three-point correlation function of $\delta \phi$. The lowest order non-vanishing contribution $\left(\mathcal{O}\left(\hbar^{2}\right)\right)$ reads

$$
\left\langle\delta \phi\left(\vec{x}_{1}, \alpha_{1}\right) \delta \phi\left(\vec{x}_{2}, \alpha_{2}\right) \delta \phi\left(\vec{x}_{3}, \alpha_{3}\right)\right\rangle=
$$




$$
\begin{aligned}
& X\left(\alpha_{3}\right) \int_{0}^{\alpha_{3}} d \alpha^{\prime}\left[\frac{B\left(\alpha^{\prime}\right)}{X\left(\alpha^{\prime}\right)}\left\langle\delta \phi_{1}\left(\vec{x}_{1}, \alpha_{1}\right) \delta \phi_{1}\left(\vec{x}_{3}, \alpha^{\prime}\right)\right\rangle\left\langle\delta \phi_{1}\left(\vec{x}_{2}, \alpha_{2}\right) \delta \phi_{1}\left(\vec{x}_{3}, \alpha^{\prime}\right)\right\rangle+\left[\vec{x}_{1} \leftrightarrow \vec{x}_{2}\right]\right] \\
& +\frac{X\left(\alpha_{3}\right)}{2 m_{P}} \int_{0}^{\alpha_{3}} d \alpha^{\prime}\left[\left\langle\delta \phi_{1}\left(\vec{x}_{1}, \alpha_{1}\right) \delta \phi_{1}\left(\vec{x}_{3}, \alpha^{\prime}\right)\right\rangle\left\langle\delta \phi_{1}\left(\vec{x}_{2}, \alpha_{2}\right) \tilde{\eta}\left(\vec{x}_{3}, \alpha^{\prime}\right)\right\rangle+\left[\vec{x}_{1} \leftrightarrow \vec{x}_{2}\right]\right] \\
& +2 \times 2 \text { terms } .
\end{aligned}
$$

The term proportional to $X\left(\alpha_{3}\right) / 2 m_{P}$ in the r.h.s. of this equation can be recast in the form

$$
\frac{X\left(\alpha_{2}\right) X\left(\alpha_{3}\right)}{2 m_{P}} \int_{0}^{\alpha_{m i n}} d \alpha^{\prime} X^{-1}\left(\alpha^{\prime}\right) \frac{H^{2}\left(\alpha^{\prime}\right)}{(2 \pi)^{2}}\left\langle\delta \phi_{1}\left(\vec{x}_{1}, \alpha_{1}\right) \delta \phi_{1}\left(\vec{x}_{3}, \alpha^{\prime}\right)\right\rangle j_{0}\left(q_{s}\left(\alpha^{\prime}\right)\left|\vec{x}_{2}-\vec{x}_{3}\right|\right)
$$

where we defined $\alpha_{\min } \equiv \min \left[\alpha_{3}, \alpha_{2}\right]$. We need now to compute the $\delta \phi$ auto-correlation function. To this aim, recalling that $\alpha$ is the time when the perturbation wavelength $\sim a / q$ equals the size of the coarse-graining domain, we change the integration variable in Eq.(19)) from $\alpha$ to $q=\sqrt{8 \pi V(\alpha) / 3} q_{*} e^{\alpha} / H_{*} m_{P}$. The subscript $*$ denotes quantities evaluated at the time when we start to solve the Langevin equation; this is chosen in such a way that the patch of the universe where we live is homogeneous on a scale slightly above our present horizon (see e.g. the discussion by Mollerach et al. 1991). We then find

$$
\left\langle\delta \phi_{1}\left(\vec{x}_{1}, \alpha_{1}\right) \delta \phi_{1}\left(\vec{x}_{3}, \alpha^{\prime}\right)\right\rangle=\frac{1}{2 \pi^{2}} \int_{q_{*}}^{q_{\min }\left(q_{1}, q^{\prime}\right)} d q q^{2} P(q) \frac{X\left(q_{1}\right) X\left(q^{\prime}\right)}{X(q) X(q)} j_{0}\left(q\left|\vec{x}_{1}-\vec{x}_{3}\right|\right)
$$

where we defined $P(q) \equiv \frac{1}{2} q^{-3} H^{2}(q)$ where $\alpha=\alpha(q)$. Using this we can rewrite Eq.(22) as

$$
\begin{aligned}
& \frac{X\left(q_{1}\right) X\left(q_{2}\right) X\left(q_{3}\right)}{2 m_{P}} \int \frac{d^{3} q^{\prime}}{(2 \pi)^{3}} \int \frac{d^{3} q}{(2 \pi)^{3}} \frac{P\left(q^{\prime}\right) P(q)}{X^{2}(q)} \\
& \quad \times W\left(q^{\prime} ; q_{\min }\left(q_{3}, q_{2}\right)\right) W\left(q ; q_{\min }\left(q_{1}, q^{\prime}\right)\right) e^{i \vec{q} \cdot\left(\vec{x}_{1}-\vec{x}_{3}\right)} e^{i \vec{q}^{\prime} \cdot\left(\vec{x}_{2}-\vec{x}_{3}\right)}
\end{aligned}
$$

where we defined the filter function $W\left(q ; q_{i}\right) \equiv \Theta\left(q-q_{*}\right)-\Theta\left(q-q_{i}\right)(\Theta$ is the Heaviside function).

A similar analysis can be performed for the term proportional to $B(\alpha)$ in Eq.(21). We get

$$
\begin{aligned}
& X\left(q_{1}\right) X\left(q_{2}\right) X\left(q_{3}\right) \int_{q_{*}}^{q_{3}} \frac{d q^{\prime}}{q^{\prime}} B\left(q^{\prime}\right) X\left(q^{\prime}\right) \int \frac{d^{3} q^{\prime \prime}}{(2 \pi)^{3}} \int \frac{d^{3} q^{\prime \prime \prime}}{(2 \pi)^{3}} \frac{P\left(q^{\prime \prime}\right)}{X^{2}\left(q^{\prime \prime}\right)} \frac{P\left(q^{\prime \prime \prime}\right)}{X^{2}\left(q^{\prime \prime \prime}\right)} \\
& \times W\left(q^{\prime \prime \prime} ; q_{\min }\left(q_{2}, q^{\prime}\right)\right) W\left(q^{\prime \prime} ; q_{\min }\left(q_{1}, q^{\prime}\right)\right) e^{i \vec{q}^{\prime \prime} \cdot\left(\vec{x}_{1}-\vec{x}_{3}\right)} e^{i \vec{q}^{\prime \prime \prime} \cdot\left(\vec{x}_{2}-\vec{x}_{3}\right)} .
\end{aligned}
$$

So far we have been working in configuration space. In order to obtain the gravitational potential at Hubble radius crossing during the Friedmann era, it is convenient to Fourier transform the coarse-grained inflaton fluctuation. One has $\delta \phi(\vec{x}, \alpha(q))=(2 \pi)^{-3} \int d^{3} k \delta \phi(\vec{k}) \Theta(q-$ $k) e^{i \vec{k} \cdot \vec{x}}$, where $\delta \phi(\vec{k})$ denotes the Fourier transform of the full scalar field at the time $\alpha(q)$. This follows from the fact that at the time $\alpha(q)$ the only modes $k$ that contribute to the coarse-grained variable are those which have already left the inflationary horizon, namely $k<q$. We can then obtain the Fourier transform $\delta \phi(\vec{k}, \alpha(q))=\delta \phi(\vec{k}) \Theta(q-k)$, and, in the limit $k \rightarrow q^{-}$(or equivalently $q \rightarrow k^{+}$, that is, when we consider the horizon-crossing time of the given scale), we simply have $\delta \phi(\vec{k}, \alpha(k))=\delta \phi(\vec{k})$. In other words, at the time $\alpha(q)$ the 
Fourier transform of the coarse-grained variable coincides with that of the full field. Using these results in Eq.(21) we finally obtain the inflaton bispectrum through

$$
\begin{aligned}
& \left\langle\delta \phi\left(\vec{k}_{1}, \alpha\left(k_{1}\right)\right) \delta \phi\left(\vec{k}_{2}, \alpha\left(k_{2}\right)\right) \delta \phi\left(\vec{k}_{3}, \alpha\left(k_{3}\right)\right)\right\rangle=(2 \pi)^{3} \delta^{3}\left(\vec{k}_{1}+\vec{k}_{2}+\vec{k}_{3}\right) P\left(k_{2}\right) P\left(k_{3}\right) \\
& \quad \times \frac{X\left(k_{1}\right)}{X\left(k_{2}\right) X\left(k_{3}\right)}\left[\frac{X^{2}\left(k_{2}\right) \Theta\left(k_{2}-k_{3}\right)+X^{2}\left(k_{3}\right) \Theta\left(k_{3}-k_{2}\right)}{2 m_{P}}+2 \int_{k_{*}}^{k_{1}} \frac{d q^{\prime}}{q^{\prime}} B\left(q^{\prime}\right) X\left(q^{\prime}\right)\right] \\
& \quad+\left\{\vec{k}_{1} \leftrightarrow \vec{k}_{2}\right\}+\left\{\vec{k}_{1} \leftrightarrow \vec{k}_{3}\right\} .
\end{aligned}
$$

\subsection{The three-point function of the gravitational potential}

We want now to compute the three-point function of the peculiar gravitational potential, $\left\langle\Phi\left(r_{0} \hat{\gamma}_{1}\right) \Phi\left(r_{0} \hat{\gamma}_{2}\right) \Phi\left(r_{0} \hat{\gamma}_{3}\right)\right\rangle$. The approximate constancy of the gauge-invariant quantity $\zeta$ outside the horizon (e.g. Bardeen, Steinhardt \& Turner 1983) allows to obtain the gravitational potential during the matter-dominated era, given the value of $\delta \phi$ during inflation. During inflation one has $\zeta(\vec{x}, \alpha) \simeq-\delta \phi(\vec{x}, \alpha) /(\partial \phi / \partial \alpha)$, which is usually interpreted as a linear relation between $\zeta$ and $\delta \phi$. However, when calculating the three-point function of $\Phi$ one cannot disregard the second-order effects coming from the fluctuations of $\partial \phi / \partial \alpha$.

Recalling that

$$
\frac{\partial \phi}{\partial \alpha}=-\frac{m_{P}^{2}}{8 \pi} \frac{V^{\prime}(\phi)}{V(\phi)} \simeq-\frac{m_{P}}{8 \pi}\left[X(\alpha)+X^{\prime}(\alpha) \delta \phi\right]
$$

one gets

$$
\zeta(\vec{x})=\frac{8 \pi}{m_{P} X(\alpha)}\left(\delta \phi(\vec{x}, \alpha)-\frac{X^{\prime}(\alpha)}{X(\alpha)} \delta \phi^{2}(\vec{x}, \alpha)\right) .
$$

This equation is expressed in configuration space; the Fourier transform of the first term inside the brackets is just $\delta \phi(\vec{k}, \alpha)$. For the second term we get a convolution of the type $\int d^{3} p \delta \phi(\vec{p}) \delta \phi(\vec{k}-\vec{p})$. For the scales of interest one has $\Phi(\vec{k}) \simeq-3 \zeta(\vec{k}) / 5$. Then, adding the two above contributions and evaluating the expression at the horizon crossing time we get

$$
\Phi(\vec{k})=\frac{24 \pi}{5 m_{P} X(\alpha(k))}\left[\delta \phi(\vec{k}, \alpha(k))-\frac{X^{\prime}(\alpha(k))}{X(\alpha(k))} \int \frac{d^{3} p}{(2 \pi)^{3}} \delta \phi(\vec{p}, \alpha(k)) \delta \phi(\vec{k}-\vec{p}, \alpha(k))\right] .
$$

From this equation we calculate

$$
\begin{aligned}
& \left\langle\Phi\left(\vec{k}_{1}\right) \Phi\left(\vec{k}_{2}\right) \Phi\left(\vec{k}_{3}\right)\right\rangle=\frac{\left(24 \pi / 5 m_{P}\right)^{3}}{X\left(k_{1}\right) X\left(k_{2}\right) X\left(k_{3}\right)}\left\langle\delta \phi\left(\vec{k}_{1}\right) \delta \phi\left(\vec{k}_{2}\right) \delta \phi\left(\vec{k}_{3}\right)\right\rangle+ \\
& \quad+\frac{\left(-24 \pi / 5 m_{P}\right)^{3} X^{\prime}\left(k_{1}\right)}{X^{2}\left(k_{1}\right) X\left(k_{2}\right) X\left(k_{3}\right)} \int \frac{d^{3} p}{(2 \pi)^{3}}\left\langle\delta \phi(\vec{p}) \delta \phi\left(\vec{k}_{1}-\vec{p}\right) \delta \phi\left(\vec{k}_{2}\right) \delta \phi\left(\vec{k}_{3}\right)\right\rangle \\
& \quad+\left\{\vec{k}_{1} \leftrightarrow \vec{k}_{2}\right\}+\left\{\vec{k}_{1} \leftrightarrow \vec{k}_{3}\right\}
\end{aligned}
$$

Using the fact that, at horizon crossing, $\left\langle\delta \phi\left(\vec{k}_{1}\right) \delta \phi\left(\vec{k}_{2}\right)\right\rangle=(2 \pi)^{3} P\left(k_{1}\right) \delta^{3}\left(\vec{k}_{1}+\vec{k}_{2}\right)$, we can write

$$
\left\langle\Phi\left(\vec{k}_{1}\right) \Phi\left(\vec{k}_{2}\right)\right\rangle=(2 \pi)^{3} \delta^{3}\left(\vec{k}_{1}+\vec{k}_{2}\right) f^{2}\left(\alpha\left(k_{1}\right)\right) P\left(k_{1}\right),
$$


with $f(\alpha(k)) \equiv 24 \pi / 5 m_{P} X(\alpha(k))$. Finally, using Eqs.(31) and (26) we find

$$
\begin{aligned}
& \left\langle\Phi\left(\vec{k}_{1}\right) \Phi\left(\vec{k}_{2}\right) \Phi\left(\vec{k}_{3}\right)\right\rangle=\left(\frac{24 \pi}{5 m_{P}}\right)^{3}(2 \pi)^{3} \delta^{3}\left(\vec{k}_{1}+\vec{k}_{2}+\vec{k}_{3}\right) \frac{P\left(k_{2}\right)}{X^{2}\left(k_{2}\right)} \frac{P\left(k_{3}\right)}{X^{2}\left(k_{3}\right)} \\
& \quad \times\left\{\frac{X^{2}\left(k_{2}\right) \Theta\left(k_{2}-k_{3}\right)+X^{2}\left(k_{3}\right) \Theta\left(k_{3}-k_{2}\right)}{2 m_{P}}-\frac{2 X^{\prime}\left(k_{1}\right) X\left(k_{2}\right) X\left(k_{3}\right)}{X^{2}\left(k_{1}\right)}+\right. \\
& \left.\quad+2 \int_{k_{*}}^{k_{1}} \frac{d q^{\prime}}{q^{\prime}} B\left(q^{\prime}\right) X\left(q^{\prime}\right)\right\}+\left\{\vec{k}_{1} \leftrightarrow \vec{k}_{2}\right\}+\left\{\vec{k}_{1} \leftrightarrow \vec{k}_{3}\right\}
\end{aligned}
$$

We are interested in considering perturbation modes that left the horizon about 60 e-foldings before the end of the inflationary epoch. In the explicit examples below $X(k)$ turns out to be a slowly varying function of $k$. We therefore approximate $X(k) \sim X_{60}$ in what follows. Then, to the lowest non-vanishing order, the three-point correlation function is

$$
\begin{aligned}
& \left\langle\Phi\left(\vec{k}_{1}\right) \Phi\left(\vec{k}_{2}\right) \Phi\left(\vec{k}_{3}\right)\right\rangle=\frac{1}{f_{60}}\left[\frac{X_{60}}{2 m_{P}}-\frac{2 X_{60}^{\prime}}{X_{60}}+\frac{2}{X_{60}} \int_{k_{*}}^{k_{60}} \frac{d q}{q} B(\alpha(q)) X(\alpha(q))\right] \\
& \quad \times(2 \pi)^{3} \delta^{3}\left(\vec{k}_{1}+\vec{k}_{2}+\vec{k}_{3}\right)\left[P_{\Phi}\left(k_{1}\right) P_{\Phi}\left(k_{2}\right)+P_{\Phi}\left(k_{2}\right) P_{\Phi}\left(k_{3}\right)+P_{\Phi}\left(k_{3}\right) P_{\Phi}\left(k_{1}\right)\right]
\end{aligned}
$$

with $f_{60} \equiv 24 \pi / 5 m_{P} X_{60}$. Using the expression for $P(q)$ we obtain the power-spectrum for the peculiar gravitational potential $P_{\Phi}(k)=2 \pi^{2} f_{60}^{2} k^{-3} H^{2}(\alpha(k)) / 4 \pi^{2} \simeq \frac{1}{2} f_{60}^{2} H_{60}^{2} k^{-3}\left(k / k_{*}\right)^{n-1}$, up to possible presence of small logarithmic corrections. The primordial spectral index $n$ is related to the inflationary parameters by the approximate relation $n \simeq 1-\left(X_{60}^{2} / 8 \pi\right)+$ $\left(m_{P} X_{60}^{\prime} / 4 \pi\right)$ (Turner 1993). Now we can obtain the two- and three-point correlation functions for $\Phi$ in configuration space by inverse Fourier transforming Eq.(31) and Eq.(33). We then get Eq.(8) and Eq.(9) with

$$
\mathcal{Q}^{2}=\frac{8 \pi^{2} H_{60}^{2}}{5 m_{P}^{2} X_{60}^{2}} \frac{\Gamma(3-n) \Gamma\left(\frac{3}{2}+\frac{n}{2}\right)}{\left[\Gamma\left(2-\frac{n}{2}\right)\right]^{2} \Gamma\left(\frac{9}{2}-\frac{n}{2}\right)}
$$

and

$$
\Phi_{3}=\frac{1}{f_{60}}\left[\frac{X_{60}}{2 m_{P}}-\frac{2 X_{60}^{\prime}}{X_{60}}+\frac{2}{X_{60}} \int_{k_{*}}^{k_{60}} \frac{d q}{q} B(\alpha(q)) X(\alpha(q))\right]
$$

In order to obtain Eq.(8) and Eq.(9), we had to use the definition of $\mathcal{C}_{\ell}$. The one given in Section 2 differs from the results obtained here because of the window function $W(k)$, appearing in the inflationary expressions; this can be however neglected if we account for the oscillating behaviour of $j_{\ell}$ for large arguments and for the fact that $j_{\ell} \rightarrow 0$ for small arguments (that helps in cancelling the lower divergence).

\section{The CMB skewness}

In what follows we will restrict our analysis to the mean skewness as given by Eq.(13), for $\alpha=0$. In the numerical calculation below we will assume that the higher multipoles are weighted by a 7.5 FWHM beam, resulting in $\sigma=3.2$ (e.g. Wright et al. 1992). To normalize 
our predictions we will consider the rms quadrupole obtained by Seljak \& Bertschinger (1993) through a Maximum-Likelihood analysis, namely $Q_{r m s-P S}=(15.7 \pm 2.6) \exp [0.46(1-n)] \mu K$ (see also Scaramella \& Vittorio 1993 and Smoot et al. 1993). P For the models with $n \lesssim 1$, considered below, this expression should be multiplied by an extra factor $[(3-n) /(14-$ $12 n)]^{1 / 2}$ to account for the effective decrease in the estimated value of $Q_{r m s-P S}$ due to the contribution of gravitational waves (Lucchin, Matarrese \& Mollerach 1992). For the mean temperature we take the FIRAS determination $T_{0}=2.726 \pm 0.01 K$ (Mather et al. 1993).

To estimate the amplitude of the non-Gaussian character of the fluctuations we will consider the "dimensionless" skewness $\mathcal{S}_{1} \equiv\left\langle C_{3}(0)\right\rangle /\left\langle C_{2}(0)\right\rangle^{3 / 2}$. Alternatively, if we want our results to be independent of the normalization, we may also define the ratio $\mathcal{S}_{2} \equiv$ $\left\langle C_{3}(0)\right\rangle /\left\langle C_{2}(0)\right\rangle^{2}$, as suggested by the hierarchical aspect of our expression for the skewness. We obtain

$$
\mathcal{S}_{1}=\frac{\sqrt{45 \pi}}{32 \pi^{2}} \mathcal{Q} X_{60}^{2}\left[1-4 m_{P} \frac{X_{60}^{\prime}}{X_{60}^{2}}+\mathcal{G}\right] \mathcal{I}_{3 / 2}(n)
$$

and

$$
\mathcal{S}_{2}=\frac{15}{16 \pi} X_{60}^{2}\left[1-4 m_{P} \frac{X_{60}^{\prime}}{X_{60}^{2}}+\mathcal{G}\right] \mathcal{I}_{2}(n)
$$

with $\mathcal{G}=4 m_{P} X_{60}^{-2} \int_{k_{*}}^{k_{60}}(d q / q) B(\alpha(q)) X(\alpha(q))$ and where we also defined the spectral indexdependent geometrical factor

$$
\mathcal{I}_{p}(n)=\frac{\frac{1}{3} \sum_{\ell_{1}, \ell_{2}, \ell_{3}}\left(2 \ell_{1}+1\right)\left(2 \ell_{2}+1\right)\left(2 \ell_{3}+1\right)\left[\mathcal{C}_{\ell_{1}} \mathcal{C}_{\ell_{2}}+\mathcal{C}_{\ell_{2}} \mathcal{C}_{\ell_{3}}+\mathcal{C}_{\ell_{3}} \mathcal{C}_{\ell_{1}}\right] \mathcal{W}_{\ell_{1}} \mathcal{W}_{\ell_{2}} \mathcal{W}_{\ell_{3}} \mathcal{F}_{\ell_{1}, \ell_{2}, \ell_{3}}}{\left[\sum_{\ell}(2 l+1) \mathcal{C}_{\ell} \mathcal{W}_{\ell}^{2}\right]^{p}}(38)
$$

where the exponent $p$ in the denominator takes values $3 / 2$ and 2 for $\mathcal{S}_{1}$ and $\mathcal{S}_{2}$, respectively. The numerical factors $\mathcal{I}_{p}(n)$ in Eq. (38) are plotted in Figure 3 for different values of the primordial index.

\subsection{Inflationary models}

Let us now specialize our general expressions to some simple inflationary models.

\section{Exponential potential}

Let us first consider power-law inflation driven by the exponential potential $V(\phi)=$ $V_{0} \exp (-\lambda \kappa \phi)$, with $\lambda<\sqrt{2}$ (Lucchin \& Matarrese 1985). In this case the power-spectrum is an exact power-law with $n=1-2 \lambda^{2} /\left(2-\lambda^{2}\right)$. We note in passing that the right spectral dependence of the perturbations can be recovered using the above stochastic approach

\footnotetext{
${ }^{3}$ Note that this value of $Q_{r m s-P S}$ assumes a multivariate Gaussian distribution function, accounting for both the signal and the noise; while in principle one should repeat the analysis consistently with the assumed statistics of the temperature perturbations, the quasi-Gaussian nature of our fluctuation field allows to extrapolate this Maximum-Likelihood estimate without sensible corrections.
} 
(Mollerach et al. 1991). For this model we find $X=-\sqrt{8 \pi} \lambda$, whose constant value implies $A=B=0$. We then have

$$
\mathcal{S}_{1}=\frac{3 \lambda}{4} \frac{H_{60}}{m_{P}} \mathcal{I}_{3 / 2}(n)\left[\frac{\Gamma(3-n) \Gamma\left(\frac{3}{2}+\frac{n}{2}\right)}{\left[\Gamma\left(2-\frac{n}{2}\right)\right]^{2} \Gamma\left(\frac{9}{2}-\frac{n}{2}\right)}\right]^{1 / 2} ; \quad \mathcal{S}_{2}=\frac{15}{2} \lambda^{2} \mathcal{I}_{2}(n)
$$

The COBE results constrain the amplitude of $H_{60}$. For the case $n=0.8$ we have $H_{60} / m_{P}=$ $1.8 \times 10^{-5}$. This gives $\mathcal{S}_{1}=9.7 \times 10^{-6}$ and $\mathcal{S}_{1}=1.1 \times 10^{-5}$, without and with the quadrupole contribution respectively, while $\mathcal{S}_{2}=1.3$ in both cases.

\section{Quartic potential}

Consider now the potential $\frac{1}{4} \lambda \phi^{4}$ for chaotic inflation (Linde 1983, 1985). For this model $X=4 m_{P} / \phi$ and therefore $m_{P} B \sim m_{P}^{2} X^{\prime \prime} \ll X$. Thus we can take $\mathcal{G} \sim 0$ in the coefficients of Eqs.(37) and (36) for the dimensionless skewness parameters. By using the slow-roll

solution for the inflaton we may write $X \simeq 4 m_{P} \phi_{60}^{-1}\left[1+\left(2 \sqrt{\frac{\lambda}{3}} / \kappa H_{60}\right) \ln \left(k / k_{60}\right)\right]$ where the logarithmic correction to the scale invariant power-spectrum is small. Inflation ends when the inflaton takes the value $\phi_{\text {end }} \simeq \sqrt{2 / 3 \pi} m_{P}$ implying $\phi_{60} \simeq \sqrt{60 / \pi} m_{P}$. In this case the spectral index is $n \simeq 1$. We find

$$
\mathcal{S}_{1}=\sqrt{\frac{2 \lambda}{\pi}} \frac{\phi_{60}}{m_{P}} \mathcal{I}_{3 / 2}(1) ; \quad \mathcal{S}_{2}=\frac{30}{\pi}\left(\frac{m_{P}}{\phi_{60}}\right)^{2} \mathcal{I}_{2}(1) .
$$

$C O B E$ constrains the value of $\lambda$ to be $\lambda \simeq 1.4 \times 10^{-13}$. We get $\mathcal{S}_{1}=5.2 \times 10^{-6}$ and $\mathcal{S}_{1}=6.0 \times 10^{-6}$ without and with the quadrupole contribution respectively, while $\mathcal{S}_{2}=0.5$.

\section{Quadratic potential}

Another simple potential for chaotic inflation is $\frac{1}{2} m_{\phi}^{2} \phi^{2}$ (Linde 1983, 1985). In this case $X \simeq 2 m_{P} \phi_{60}^{-1}\left[1+\left(2 / \kappa^{2} \phi_{60}^{2}\right) \ln \left(k / k_{60}\right)\right], \phi_{60} \simeq \sqrt{30 / \pi} m_{P}$ and $n \simeq 1$. We find

$$
\mathcal{S}_{1}=\frac{3}{2 \sqrt{\pi}} \frac{m_{\phi}}{m_{P}} \mathcal{I}_{3 / 2}(1) ; \quad \mathcal{S}_{2}=\frac{45}{4 \pi}\left(\frac{m_{P}}{\phi_{60}}\right)^{2} \mathcal{I}_{2}(1)
$$

with $m_{\phi} / m_{P} \simeq 1.1 \times 10^{-6}$ from the $C O B E$ normalization. We get $\mathcal{S}_{1}=3.9 \times 10^{-6}$ and $\mathcal{S}_{1}=4.5 \times 10^{-6}$ without and with the quadrupole contribution respectively, while $\mathcal{S}_{2}=0.4$.

\section{Hybrid inflation model}

Finally, let us consider a model of hybrid inflation recently proposed by Linde (1993). Inflation happens in this model during the slow-roll evolution of the inflaton in the effective potential $V(\phi)=V_{0}+\frac{1}{2} m^{2} \phi^{2}$, where $V_{0}=M^{4} / 4$ is a cosmological-constant-like term. At a given time, when the inflaton field takes the value $\phi=M$, its coupling with a second scalar field triggers a second-order phase transition of the latter (whose vacuum energy density is responsible for the cosmological constant term), which makes inflation end. An interesting prediction of this model is that the spectral index of density fluctuations for wavelengths 
which left the horizon while $V_{0}>m^{2} \phi^{2} / 2$ is larger than unity, namely $n \simeq 1+2 m^{2} / \kappa^{2} V_{0}$ (Mollerach et al. 1993). For this potential we find $X=m_{P} m^{2} \phi / V_{0}$, implying $\mathcal{G}=0$. We then obtain

$$
\begin{aligned}
& \mathcal{S}_{1}=\frac{\sqrt{3}}{2} \frac{\phi_{60}}{m_{P}} \frac{m^{2}}{M^{2}}\left(1-\frac{M^{4}}{m^{2} \phi_{60}^{2}}\right) \mathcal{I}_{3 / 2}(n)\left[\frac{\Gamma(3-n) \Gamma\left(\frac{3}{2}+\frac{n}{2}\right)}{\left[\Gamma\left(2-\frac{n}{2}\right)\right]^{2} \Gamma\left(\frac{9}{2}-\frac{n}{2}\right)}\right]^{1 / 2} \\
& \mathcal{S}_{2}=\frac{15}{\pi} \frac{m_{P}^{2} m^{4} \phi_{60}^{2}}{M^{8}}\left(1-\frac{M^{4}}{m^{2} \phi_{60}^{2}}\right) \mathcal{I}_{2}(n)
\end{aligned}
$$

The slow-roll solution for the inflaton gives $\phi_{60}=M \exp \left[60 \mathrm{~m}^{2} / \kappa^{2} V_{0}\right]$. Choosing a value $n=1.1$, the $C O B E$ results constrain the free parameters to be $M \simeq 1.3 \times 10^{-4} m_{P}$ and $m \simeq 10^{-8} m_{P}$. In this case we find $\mathcal{S}_{1}=-1.1 \times 10^{-5}$ and $\mathcal{S}_{1}=-1.3 \times 10^{-5}$ without and with the quadrupole contribution, while $\mathcal{S}_{2}=-1.6$. Note that these results suggest some correlation between the sign of the skewness and the value of the spectral index $n$.

\section{Conclusions}

The results reported in the previous section seem to preclude any chance of actually obtaining observable non-Gaussian signals at least in the frame of inflation, unless one resorts to more complicated multiple-field models (e.g. Allen, Grinstein \& Wise 1987; Kofman et al. 1991; Salopek 1992). However, it should be stressed that all the results reported in Section 2 would apply to any large-scale anisotropy where the temperature fluctuation can be obtained by a local perturbative calculation (this is not the case, for instance, for secondary anisotropies, which are to be ascribed to an integrated effect): under these conditions the hierarchical form of Eq.(9), where the bispectrum is a sum of products of two power-spectra, holds.

One may also wonder whether the general analysis of the CMB skewness generated in the frame of the inflationary model may provide further constraints on the model parameters. We did not address this issue in the present paper, where we only considered some simple realistic models. In these cases a relevant constraint can be obtained. Both for the exponential potential and for the polynomial ones the skewness can be estimated by taking just the first term in the brackets of Eqs.(36) and (37). An upper limit on its magnitude is then obtained by requiring that they give rise to an accelerated universe expansion (i.e. inflation), which provides a constraint on the steepness of the potential, $X^{2}<24 \pi$. This corresponds to $\mathcal{S}_{1} \lesssim 10^{-4}$ and $\mathcal{S}_{2} \lesssim 20$.

Notwithstanding the non-zero value of the skewness, any actual detection of this signal is hardly distinguishable from the cosmic variance noise in which it is embedded: the intrinsic limitation induced by our impossibility of making measurements in more than one universe. In fact, the overall coefficient of $\mathcal{S}_{1}$ is generically much smaller than the dimensionless $r m s$ skewness calculated from an underlying Gaussian density field. Removing the quadrupole contribution to reduce the cosmic rms skewness, as recently suggested by Luo (1993b), does not change this main conclusion, because the predicted mean skewness would also be reduced by a comparable factor. Things get even worse if we take into account that no sampling of the CMB sky is complete, not even the COBE one, where a cut for galactic latitudes 
$|b|<20^{\circ}$ is required. As shown by Scott et al. (1993), this "sample variance" contributes in hiding the signal. Therefore, any possible skewness detection on the COBE scale is most probably due to these statistical effects than to any primordial non-Gaussian feature.

Acknowledgement: The work of A. Gangui was supported in part by the Italian MAE. F. Lucchin and S. Matarrese acknowledge financial support from the Italian MURST. The work of S. Mollerach was supported by a grant from the Commission of European Communities (Human capital and mobility programme).

\section{References}

Abbott, L.F. \& Wise, M.B. 1984, ApJ, 282, L47

Allen, T.J., Grinstein, B. \& Wise, M.B. 1987, Phys. Lett. B, 197, 66

Bardeen, J.M., Steinhardt, P.J. \& Turner, M.S. 1983, Phys. Rev., D28, 679

Barrow, J.D. \& Coles, P. 1990, MNRAS, 244, 188

Bond, J.R. \& Efstathiou, G. 1987, MNRAS, 226, 655

Coulson, D., Ferreira, P., Graham, P. \& Turok, N. 1993, preprint (PUP-TH-1429)

Coulson, D., Pen, U.-L. \& Turok, N. 1993, preprint (PUP-TH-1393)

Fabbri, R., Lucchin, F. \& Matarrese, S. 1987, ApJ, 315, 1

Falk, T., Rangarajan, R. \& Srednicki, M. 1993, ApJ, 403, L1

Goncharov, A.S., Linde, A.D. \& Mukhanov, V.F. 1987, Int. J. Mod. Phys., A2, 561

Graham, P., Turok, N., Lubin, P.M. \& Schuster, J.A. 1993, preprint (PUP-TH-1408)

Hinshaw, G. et al. 1993, ApJ, submitted (preprint astro-ph/9311030)

Kofman, L.K., Blumenthal, G.R., Hodges, H. \& Primack, J.R. 1990, in Proc. Workshop on Large-Scale Structures and Peculiar Motions in the Universe, Rio de Janeiro, May 1989, Latham, D.W. \& da Costa, L.N. eds., ASP Conference Series, Vol. 15

Linde, A. 1983, Phys. Lett. B, 129, 177

Linde, A. 1985, Phys. Lett. B, 162, 281

Linde, A. 1993, preprint (astro-ph/9307002)

Lucchin, F. \& Matarrese, S. 1985, Phys. Rev., D32, 1316 
Lucchin, F., Matarrese, S. \& Mollerach, S. 1992, ApJ, 401, L49

Luo, X. 1993a, Phys. Rev. D, submitted (FNAL-PUB-93/294-A)

Luo, X. 1993b, ApJ, submitted (preprint astro-ph/9312004)

Luo, X. \& Schramm, D.N. 1993, Phys. Rev. Lett., 71, 1124

Martínez-González, E., Sanz, J.L. \& Silk, J. 1992, Phys. Rev. D46, 4193

Mather, J. et al. 1993, ApJ, in press

Messiah, A. 1976, Quantum Mechanics, Vol.2 (Amsterdam: North-Holland)

Moessner, R., Perivolaropoulos, L. \& Brandenberger, R. 1993 preprint (BROWN-HET-911)

Mollerach, S., Matarrese, S. \& Lucchin, F. 1993, ApJ, submitted (preprint astro-ph/9309054)

Mollerach, S., Matarrese, S., Ortolan, A. \& Lucchin, F. 1991, Phys. Rev. D44, 1670

Rees, M. \& Sciama, D.W. 1968, Nature, 217, 511

Sachs, R. \& Wolfe, A. 1967, ApJ, 147, 73

Salopek, D.S. 1992, Phys. Rev. D45, 1005

Scaramella, R. \& Vittorio, N. 1991, ApJ, 375, 439

Scaramella, R. \& Vittorio, N. 1993 MNRAS, 263, L17

Scott, D., Srednicki, M. \& White, M. 1993, ApJ, submitted (preprint astro-ph/9305030)

Seljak, U. \& Bertschinger, E. 1993, ApJ, 417, L9

Smoot, G.F. et al. 1992, ApJ, 396, L1

Smoot, G.F. et al. 1993, ApJ, submitted (preprint astro-ph/9312031)

Srednicki, M. 1993, ApJ, 416, L1

Starobinskii, A. 1986, in Field Theory, Quantum Gravity and Strings, Proceedings of the Seminar Series, Meudon and Paris, France, 1984-1985, de Vega, H.J. \& Sanchez, N. eds., Lecture Notes in Physics Vol. 246 (Berlin: Springer-Verlag)

Turner, M.S. 1993, Phys. Rev. D48, 3502

Wright, E.L. et al. 1992, ApJ, 396, L13

Yi, I. \& Vishniac, E.T. 1993, Phys. Rev. D48, 950 


\section{Figure captions}

Figure 1: Collapsed three-point function (with and without the quadrupole contribution), normalized to the skewness, as a function of the angular separation $\alpha$ (left panel). The different curves refer to three typical values of the primordial spectral index: $n=0.8,1,1.2$. The symbols in the left panel are the same as in the right one, which shows a vertical expansion of the same plot.

Figure 2: Normalized rms skewness of a Gaussian temperature fluctuation field as a function of the spectral index $n$, both including and removing the quadrupole contribution.

Figure 3: Geometrical factors $\mathcal{I}_{p}(n)$ as a function of $n$. Crosses are for $\mathcal{I}_{3 / 2}(n)$, where the quadrupole term is removed. Diamonds correspond to $\mathcal{I}_{3 / 2}(n)$ including the quadrupole contribution. Open boxes correspond to $\mathcal{I}_{2}(n)$. For the latter case the difference in including or removing the quadrupole contribution is $\mathcal{O}\left(10^{-2}\right)$, which is not perceivable in this graph. 